\title{
An Analytical Framework for Disconnection Prediction in Wireless Networks
}

\author{
Gitanjali Bhutani \\ WCDMA, Alcatel-Lucent Technologies India Private Limited, Bangalore, India \\ Email: gitanjali.bhutani@alcatel-lucent.com
}

Received 5 May 2014; revised 25 May 2014; accepted 4 June 2014

Copyright (C) 2014 by author and Scientific Research Publishing Inc. This work is licensed under the Creative Commons Attribution International License (CC BY). http://creativecommons.org/licenses/by/4.0/

c) (i) Open Access

\begin{abstract}
The stability and reliability of links in wireless networks is dependent on a number of factors such as the topology of the area, inter-base station or inter-mobile station distances, weather conditions and so on. Link instability in wireless networks has a negative impact on the data throughput and thus, the overall quality of user experience, even in the presence of sufficient bandwidth. An estimation of link quality and link availability duration can drastically increase the performance of these networks, allowing the network or applications to take proactive measures to handle impending disconnections. In this paper we look at a mathematical model for predicting disconnection in wireless networks. This model is originally intended to be implemented in base stations of cellular networks, but is independent of the wireless technology and can thus be applied to different types of networks with minimum changes.
\end{abstract}

\section{Keywords}

Wireless Networks, Mobility Modeling, Disconnection Prediction

\section{Introduction}

As the world gears towards the adoption of $4 \mathrm{G}$ mobile networks, we are going to see one of the largest deployments of an advanced mobile technology. However, there are many issues that need attention for such a large deployment. Base stations support a limited number of physical connections. When a mobile moves from one base station to another, if the destination base station is already servicing the maximum possible number of mobiles, the call would be dropped. Prediction of the mobility and the approximate time of being under the coverage area of the current base station will allow resource allocation in advance, thus preventing call drops. Towards this end, mobility management and disconnection prediction schemes are important, as they enable seamless hand- overs and hence, an improved quality of user experience. 
In single-hop wireless networks like GSM and UMTS, prediction of disconnection can allow the base station to cache TCP data and acknowledgements for packet connections to the mobile. This helps prevent loss of throughput when the connection to the mobile is restored. Disconnection prediction schemes to prevent TCP's congestion control mechanisms from kicking in and causing a drastic reduction in throughput are discussed in [1] and [2]. In the Freeze TCP scheme described in [1], the mobile indicates its incapability to receive data to the content server just prior to the disconnection so that no data is lost during the period of the disconnection. In the TCP ACK Pacing scheme described in [2] the base station spaces out the TCP acknowledgements that are sent to the server based on the prediction information, thus, keeping the server entirely transparent to the disconnection.

In this paper, we look at the Gauss-Markov mobility-modeling scheme developed in [3] and try to apply it to our problem of predicting the disconnection duration of a mobile. The first portion of the paper deals with the application of the original Gauss-Markov mobility model for the disconnection prediction problem. However, we realize that such an application leads us to a mathematical expression for disconnection duration that may be difficult to calculate in field applications. Hence, we try to turn the original Gauss-Markov model on its head in our search for a more tractable expression for disconnection duration period.

\section{Related Work}

In this section, we first look at the most popular mobility models in use today. We then briefly discuss the work done in the area of disconnection prediction.

\subsection{Mobility Modeling}

The need for testing of networks in realistic conditions gives rise to the concept of mobility modeling. There are two types of mobility models that are used in the simulation of networks: a) traces and b) synthetic mobility models. Traces are mobility patterns observed in real life systems. Traces provide accurate information especially if they involve a large number of users and the observations are carried out over long time periods. However, for newer network environments, traces are not yet available and in this case synthetic mobility models are used. Synthetic models attempt to realistically represent the behavior of mobile nodes without the use of traces. The synthetic mobility models can further be categorized into entity and group mobility models. Entity mobility models are those in which the movement of each mobile node is independent of the other. Group mobility models are those in which the movement of mobile nodes is inter-dependent.

The Random Walk mobility model described in [4] has become the foundation of a number of mobility models. In this model, each node selects a direction to move in which is in a specified range. The node chooses its speed based on a user-defined distribution of speeds and then moves in the chosen direction with the chosen speed. After some randomly chosen amount of time, each node halts and selects a new direction to move in. This is a memoryless mobility model where the direction and speed of the node at any point is independent of its speed and movement before this point. This characteristic generates unrealistic movements such as sudden stops and sharp turns. The Random Direction Model discussed in [5] is a variation of this, where instead of stopping after some amount of time, each node moves till it reaches the boundary of the simulation area and then chooses a new direction to move in. This model aims at maintaining a constant density of nodes throughout the simulation. An evaluation of this model shows that network partitions are more likely with this mobility model than others. Also, since, the nodes travel to and then pause at the end of the simulation area, the average hop count data packets in this mobility model is higher than the hop count of other mobility models. In [6] and [7] a different variation of this model is proposed. In this case, when the node reaches the boundary of the simulation area, it is reflected back into the simulation area while the velocity of the node is held constant.

The Gauss Markov Mobility model eliminates the disadvantage of the Random models in terms of these being memoryless models. In this model, the speed and direction of the model at time $\mathrm{n}$ depends on its speed and direction at time $(n-1)$ and a random variable. To ensure that a node does not remain near an edge of the grid for a long period of time, the nodes are forced away from an edge when they move within a certain distance of the edge. The advantages of using a Gauss-Markov model instead of the random-walk mobility model are highlighted in [3]. In the real world users generally move with a particular destination in mind and hence, their location in the future is a function of their current location and velocity. This information is better represented by using a Gauss Markov model as compared to the random-walk model which is memoryless. Simulations per- 
formed show that this scheme provides a performance improvement ranging from unity to a factor of 10 in comparison to the regular non-predictive distance based schemes.

\subsection{Wireless Link Status Prediction}

Most of the work on link status prediction uses artificial intelligence based schemes to predict disconnection. One such scheme for link quality prediction and link estimation called 4C [8] uses previously collected link quality data to construct three different machine-learning models: Native Bayes classifier, logistic regression and artificial neural networks. These models are constructed based on a combination of packet reception rate (PRR) for link estimation and Received Signal Strength Indicator (RSSI), Link Quality Input (LQI) and Signal to Noise Ratio (SNR). The output of each model is the success probability of delivering each packet. The authors compare the prediction accuracy of each of these models against a Bernoulli process whose success probability is set to the packet reception rate. Experimental results show that all three models have a greater prediction accuracy than the Bernoulli process with the Logistic regression model having the best accuracy at very low computational cost.

In order to predict wireless network connectivity, that is, the signal to noise ratio for a mobile station, [9] proposes the use of a new Taylor Kriging model, which is basically the Kriging model with third order Taylor expansion for prediction. The prediction accuracy is compared against that of a predictor built using the Ordinary Kriging model [10] and an artificial neural network based predictor [11]. The prediction accuracy of the Taylor Kriging model is significantly higher than both these models, but the prediction error is still substantially high.

\section{Disconnection Duration Prediction for a Mobile}

In order to achieve the various advantages of seamless communication in the presence of mobility, it is important to be able to predict not only the location of the mobile, but also an impending disconnection and its duration. In this section, we look at a disconnection prediction scheme that uses the Gauss-Markov mobility model discussed in [3]. We arrive at an expression that can be used by the base station to predict the disconnection duration of a mobile.

For developing the disconnection prediction model in this paper, we make assumptions similar to those made by [3]. First, we assume that time is divided into discrete intervals, denoted by subscripts $1,2,3, \cdots, n, \cdots$ on various variables. Mobile user-equipments have the capability to measure their location $(S)$ and velocity $(v)$ in respect to the closeby base-stations. Further, we assume that the velocity $v$ of the mobile user-equipment follows a stationary Gauss-Markov process. In [3], the authors note that this is a reasonable assumption, and holds true in various practical scenarios. Accordingly, the velocity of the mobile at time $n$ can be written as

$$
v_{n}=\alpha \cdot v_{n-1}+(1-\alpha) \cdot \mu+\sqrt{1-\alpha^{2}} \cdot x_{n-1}
$$

where $\mu$ is the asymptotic mean of $v_{n}$ as $n \rightarrow \infty$. Let $\sigma^{2}$ denote the variance of $v_{n}$ as $n \rightarrow \infty ; x$ 's denote independent, uncorrelated and stationary Gaussian processes with mean $\mu_{x}=0$ and $\sigma_{x}=\sigma$. For our current analysis, we restrict ourselves to the single-dimensional case. The more realistic two-dimensional case can be derived in a similar fashion. We also restrict ourselves to a single base-station case for the purpose of analysis in this paper. We let the origin denote the location of the base-station. The mobile is assumed to have a starting location of $S_{0}$ and initial velocity of $v_{0}$. The range of the base-station is assumed to be between $-d$ and $+d$. The mobile is assumed to be disconnected outside this range.

The following can be inferred from Equation (1)

$$
\begin{aligned}
v_{1} & =\alpha \cdot v_{0}+(1-\alpha) \cdot \mu+\sqrt{1-\alpha^{2}} \cdot x_{0} \\
v_{2} & =\alpha \cdot v_{1}+(1-\alpha) \cdot \mu+\sqrt{1-\alpha^{2}} \cdot x_{1} \\
& =\alpha\left[\alpha \cdot v_{0}+(1-\alpha) \cdot \mu+\sqrt{1-\alpha^{2}} \cdot x_{0}\right]+(1-\alpha) \cdot \mu+\sqrt{1-\alpha^{2}} \cdot x_{1} \\
& =\alpha^{2} v_{0}+\left(1-\alpha^{2}\right) \cdot \mu+\sqrt{1-\alpha^{2}} \cdot\left(x_{1}+\alpha \cdot x_{0}\right)
\end{aligned}
$$




$$
v_{n}=\alpha^{n} v_{0}+\left(1-\alpha^{n}\right) \cdot \mu+\sqrt{1-\alpha^{2}} \cdot \sum_{i=0}^{n-1} \alpha^{n-i-1} x_{i}
$$

The location of the mobile after $n$ time periods can be defined as

$$
\begin{aligned}
S_{n} & =S_{0}+\sum_{i=0}^{n-1} v_{i} \\
& =S_{0}+v_{0}+\sum_{i=1}^{n-1}\left[\alpha^{i} v_{0}+\left(1-\alpha^{i}\right) \mu+\sqrt{1-\alpha^{2}} \sum_{j=0}^{i-1} \alpha^{i-j-1} x_{j}\right] \\
& =S_{0}+v_{0}\left[1+\sum_{i=1}^{n-1} \alpha^{i}\right]+\mu\left[\sum_{i=1}^{n-1}\left(1-\alpha^{i}\right)\right]+\sqrt{1-\alpha^{2}}\left[\sum_{i=1}^{n-1} \sum_{j=0}^{i-1} \alpha^{i-j-1} x_{j}\right]
\end{aligned}
$$

Let us define

$$
\begin{aligned}
& \mathcal{A} \equiv 1+\sum_{i=1}^{n-1} \alpha^{i}=\frac{\left(1-\alpha^{n}\right)}{(1-\alpha)} \\
& \mathcal{B} \equiv \sum_{i=1}^{n-1}\left(1-\alpha^{i}\right)=(n-1)-\sum_{i=1}^{n-1} \alpha^{i}=(n-1)-\frac{\alpha \cdot\left(1-\alpha^{n-1}\right)}{(1-\alpha)} \\
& \mathcal{C}=\sum_{i=1}^{n-1} \sum_{j=0}^{i-1} \alpha^{i-j-1} x_{j}
\end{aligned}
$$

So that

$$
S_{n}=S_{0}+v_{0} \cdot \mathcal{A}+\mu \cdot \mathcal{B}+\sqrt{1-\alpha^{2}} \cdot \mathcal{C}
$$

The terms of summation in calculation of $\mathcal{C}$ can be swapped. The nested summation over $j$ in Equation (12) can be swapped out so that

$$
\begin{aligned}
\mathcal{C} & =\sum_{j=0}^{n-2} \frac{x_{j}}{\alpha^{j}} \cdot \sum_{i=j+1}^{n-1} \alpha^{i-1}=\sum_{j=0}^{n-2} \frac{x_{j}}{\alpha^{j}} \cdot \sum_{i=j}^{n-2} \alpha^{i}=\sum_{j=0}^{n-2} \frac{x_{j}}{\alpha^{j}} \cdot \alpha^{j} \cdot \sum_{i=0}^{n-2-j} \alpha^{i} \\
& =\sum_{j=0}^{n-2} x_{j} \cdot \frac{\left(1-\alpha^{n-1-j}\right)}{(1-\alpha)}=\frac{1}{(1-\alpha)} \sum_{j=0}^{n-2} x_{j} \cdot\left(1-\alpha^{n-1-j}\right)
\end{aligned}
$$

Hence, location of the mobile aftern $n$ time periods, $S_{n}$ can be written as

$$
S_{n}=S_{0}+\frac{\left(1-\alpha^{n}\right)}{(1-\alpha)} v_{0}+\left[(n-1)-\frac{\alpha\left(1-\alpha^{n-1}\right)}{(1-\alpha)}\right] \mu+\frac{\sqrt{1-\alpha^{2}}}{(1-\alpha)} \sum_{j=0}^{n-2} x_{j}\left(1-\alpha^{n-1-j}\right)
$$

We now define $\mathcal{C}_{1}, \mathcal{C}_{2}$ and $\mathcal{C}_{3}$ as

$$
\mathcal{C}_{1}=\sum_{j=0}^{n-2} x_{j}\left(1-\alpha^{n-1-j}\right)=\sum_{j=0}^{n-2} X_{j}-\sum_{j=0}^{n-2} \alpha^{n-1-j} x_{j}=\mathcal{C}_{2}+\mathcal{C}_{3}
$$

Since $x_{j}$ 's are independent, uncorrelated and stationary gaussian processes with $\mu_{x}=0$ and $\sigma_{x}=\sigma, \mathcal{C}_{2}$ can be shown to be a stationary gaussion process with mean $\mu_{\mathcal{C}_{2}}=0$ and variance $\sigma_{\mathcal{C}_{2}}=(n-2) \sigma^{2}$. Mean and variance of $\mathcal{C}_{3}$ can be expressed as

$$
\begin{aligned}
& \mu_{\mathcal{C}_{3}}=\sum_{j=0}^{n-2} \alpha^{n-1-j} \mu_{x_{j}}=0 \\
& \sigma_{\mathcal{C}_{3}}^{2}=\sum_{j=0}^{n-2}\left(\alpha^{n-1-j}\right)^{2} \cdot \sigma_{x_{j}}^{2}=\sigma^{2} \sum_{j=0}^{n-2}\left(\alpha^{n-1-j}\right)^{2}=\alpha^{2} \sigma^{2}\left[\frac{1-\left(\alpha^{2}\right)^{n-1}}{1-\alpha^{2}}\right]
\end{aligned}
$$


Mean and variance of $\mathcal{C}_{1}$ can be calculated as

$$
\begin{aligned}
& \mu_{\mathcal{C}_{1}}=\mu_{\mathcal{C}_{2}}+\mu_{\mathcal{C}_{3}} \\
& \sigma_{\mathcal{C}_{1}}^{2}=\sigma_{\mathcal{C}_{2}}^{2}+\sigma_{\mathcal{C}_{3}}^{2}=(n-2) \sigma^{2}+\frac{\sigma^{2} \alpha^{2}}{\left(1-\alpha^{2}\right)}\left[1-\alpha^{2(n-1)}\right]
\end{aligned}
$$

We denote the mean and variance of $S_{n}$ by $\eta$ and $\tau^{2}$ respectively. Then

$$
\eta=S_{0}+\frac{\left(1-\alpha^{n}\right) v_{0}}{(1-\alpha)}+\left[(n-1)-\frac{\alpha\left(1-\alpha^{n-1}\right)}{(1-\alpha)}\right] \mu
$$

and

$$
\begin{aligned}
\tau^{2} & =\frac{\left(1-\alpha^{2}\right)}{(1-\alpha)^{2}}\left[(n-2) \sigma^{2}+\frac{\sigma^{2} \alpha^{2}}{\left(1-\alpha^{2}\right)}\left(1-\alpha^{2(n-1)}\right)\right] \\
& =\frac{1}{(1-\alpha)^{2}}\left[(n-2) \sigma^{2}\left(1-\alpha^{2}\right)+\sigma^{2} \alpha^{2}\left(1-\alpha^{2(n-1)}\right)\right] \\
& =\frac{\sigma^{2}}{(1-\alpha)^{2}}\left[(n-2)\left(1-\alpha^{2}\right)+\alpha^{2}-\alpha^{2 n}\right]
\end{aligned}
$$

Note that the mobile is connected at time $n$ if $-d \leq S_{n}<+d$. We denote this event of being connected by $\mathcal{S}(n)$, and that of being disconnected by $\mathcal{F}(n)$. Then

$$
\text { Prob. }(\mathcal{S}(n))=\text { Prob. }\left(-d \leq S_{n}<+d\right)=\Phi\left(\frac{(d-\eta)}{\tau}\right)-\Phi\left(\frac{(-d-\eta)}{\tau}\right)
$$

where $\Phi$ denotes the cumulative distribution function of the standard normal distribution.

$$
\text { Prob. }(\mathcal{F}(n))=1-\operatorname{Prob} .(\mathcal{S}(n))
$$

Let us denote the random variable for disconnection duration using the symbol $\Delta$. Let $\mathcal{G}(\zeta, \delta)$ denote the probability that the mobile stays connected till time $\zeta$, and then stays disconnected for exactly $\delta$ period of time. In terms of Bernoulli trials, this equates to having $(\zeta-1)$ successes $(\mathcal{S})$, followed by $\delta$ failures $(\mathcal{F})$, followed by a success $(\mathcal{S})$. Then

$$
\operatorname{Prob} .(\Delta=\delta)=\sum_{\forall \zeta} \mathcal{G}(\zeta, \delta)
$$

and

$$
\begin{aligned}
\mathcal{G}(\zeta, \delta)= & \prod_{i=1}^{\zeta-1}\left\{\Phi\left(\frac{d-\eta(i)}{\tau(i)}\right)-\Phi\left(\frac{-d-\eta(i)}{\tau(i)}\right)\right\} \times \prod_{i=\zeta}^{\zeta+\delta-1}\left\{1-\left[\Phi\left(\frac{d-\eta(i)}{\tau(i)}\right)-\Phi\left(\frac{-d-\eta(i)}{\tau(i)}\right)\right]\right\} \\
& \times\left\{\Phi\left(\frac{d-\eta(\zeta+\delta)}{\tau(\zeta+\delta)}\right)-\Phi\left(\frac{-d-\eta(\zeta+\delta)}{\tau(\zeta+\delta)}\right)\right\}
\end{aligned}
$$

Correspondingly, the probability of disconnection duration for the mobile taking a value of $\delta$ can be stated as

$$
\begin{aligned}
\text { Prob. }(\Delta=\delta)= & \sum_{\forall \zeta} \prod_{i=1}^{\zeta-1}\left\{\Phi\left(\frac{d-\eta(i)}{\tau(i)}\right)-\Phi\left(\frac{-d-\eta(i)}{\tau(i)}\right)\right\} \times \prod_{i=\zeta}^{\zeta+\delta-1}\left\{1-\left[\Phi\left(\frac{d-\eta(i)}{\tau(i)}\right)-\Phi\left(\frac{-d-\eta(i)}{\tau(i)}\right)\right]\right\} \\
& \times\left\{\Phi\left(\frac{d-\eta(\zeta+\delta)}{\tau(\zeta+\delta)}\right)-\Phi\left(\frac{-d-\eta(\zeta+\delta)}{\tau(\zeta+\delta)}\right)\right\}
\end{aligned}
$$


Note that while Equation (30) gives us a method to find the probability of disconnection duration taking on a particular value, it is difficult to calculate in practice as the summations being carried out are on possible values of $\zeta$, the starting point of the disconnection duration. To overcome this difficulty, we approach the problem through a different tack in the next section.

\section{Changing the Interpretation of the Gauss-Markov Process}

The Gauss-Markov mobility model, and even the one proposed by [3], assumes that time increments in terms of discrete periods, and the decisions about the velocity and the direction of motion are made at the transition of these time periods. While such a model is useful for other purposes, its usage complicates analysis when trying to model the path of a mobile as it moves from within-range to out-of-range and then comes back within-range. In particular, the problem posed is that the mobile can make different sized distance steps in different timeperiods, thereby making the computation of the time when it is going to land back within-range convoluted. Therefore, henceforth in this analysis we assume that location varies as a discrete variable taking on only unit positive and unit negative values based on the direction of motion, while the time taken between two location steps varies continuously based on the magnitude of velocity. Also, changes need to be done to Equation (1), where we model the inverse of velocity rather than velocity itself as a Gauss-Markov process.

$$
v_{n}=\alpha \cdot v_{n-1}+(1-\alpha) \cdot \mu+\sqrt{1-\alpha^{2}} \cdot \xi_{n-1}
$$

Based on such a model, we now try and count the number of ways in which the mobile can make forward and backward movements once it has reached the range boundary. For the mobile to take $(2 n+2)$ steps to return back to within-range, it must make $(n+1)$ forward and $(n+1)$ backward steps. These $(n+1)$ forward and $(n+1)$ backward steps can be arranged in any order, as long as the mobile does not land up within-range at any intermediate time during those $(2 n+2)$ steps. For setting up the calculation, note that the mobile must first make a forward $1 \mathrm{~F}$ step, and at the end make a backward $1 \mathrm{~B}$ step. In the meanwhile, it can at best return back to within 1-step of the starting point, but no further. Hence, for our calculations, we assume that the 1-step away point is origin, and that the mobile makes $1 \mathrm{~F}$ step to reach origin at the start of the sequence, makes a series of intermediate steps and returns back to the origin at the end of $2 n$ steps, and then makes 1B step to return to within range. We let $\{n\}$ denote the sequence of intermediate steps that the mobile makes, where it at best returns back to origin and no further. Note that in this setup, the minimum number of times the mobile can visit the origin in the meanwhile is 0 , while the maximum number of times it can do so is $(n-1)$. We work out examples of $\{n\}$ with $n=(3,4,5)$ here in Table 1, Table 2 and Table 3.

A close examination of the examples worked out in Table 1, Table 2 and Table 3 leads to the discovery of a structure. For defining this structure, we define the following operators:

Definition 1 We define unary operator $\|\cdot\|$, such that given a sequence of steps $A,\|A\|$ denotes the number of sequences contained in $A$.

Definition 2 We define binary operator $\rightarrow$, such that given two sequence of steps $A$ and $B, A \rightarrow B$ indicates the set of steps in A followed by set of steps in $B$.

Definition 3 We define $\{n, k\}$ as sequences involving $n$ forward and $n$ backward steps where the mobile returns back to origin exactly $k$ times

Theorem 1 The application of operator $\rightarrow$ generates sequences such that $\|A \rightarrow B\|=\|A\| \cdot\|B\|$.

Proof 1 As per Definition 2, $A \rightarrow B$ comprises of all sequences of $A$ followed by all sequences of $B$. Hence,

$$
A \rightarrow B \equiv A \times B
$$

where $A \times B$ denotes the set of sequences produced as a results of taking a set cartesian product of sequences of $A$ with those of $B$. Hence,

$$
\|A \rightarrow B\|=\|A \times B\|=\|A\| \cdot\|B\|
$$

Corollary 1 Let $i$ denote the first position at which the mobile returns back to origin in a $\{n, k\}$ sequence, $i \leq k$. Then $\{n, k\} \equiv(1 F \rightarrow\{i-1\} \rightarrow 1 B \rightarrow\{n-i, k-1\})$. This is so as the mobile returns back to the origin exactly after first $i$ steps, which is the same as our original problem; and having returned back to origin after $i$ steps, the mobile needs to visit the origin $k-1$ times in the rest $n-i$ steps. 
Table 1. $\{3\}$ : Series of forward and backward steps to return within-range in $(2 n+2)=8$ steps.

\begin{tabular}{cc}
\hline Number of visits to origin & Sequence of steps \\
\hline 0 & $(3 \mathrm{~F}-3 \mathrm{~B})(2 \mathrm{~F}-1 \mathrm{~B}-1 \mathrm{~F}-2 \mathrm{~B})$ \\
1 & $(1 \mathrm{~F}-1 \mathrm{~B}-2 \mathrm{~F}-2 \mathrm{~B})(2 \mathrm{~F}-2 \mathrm{~B}-1 \mathrm{~F}-1 \mathrm{~B})$ \\
2 & $(1 \mathrm{~F}-1 \mathrm{~B}-1 \mathrm{~F}-1 \mathrm{~B}-1 \mathrm{~F}-1 \mathrm{~B})$ \\
\hline
\end{tabular}

Table 2. $\{4\}$ : Series of forward and backward steps to return within-range in $(2 n+2)=10$ steps.

\begin{tabular}{cc}
$\begin{array}{c}\text { Number of visits to } \\
\text { origin } \\
0\end{array}$ & Sequence of steps \\
1 & $(1 \mathrm{~F} \rightarrow\{3\} \rightarrow 1 \mathrm{~B})$ \\
& $(1 \mathrm{~F}-1 \mathrm{~B}-1 \mathrm{~F} \rightarrow\{2\} \rightarrow 1 \mathrm{~B})(1 \mathrm{~F} \rightarrow 1 \rightarrow 1 \mathrm{~B}-1 \mathrm{~F} \rightarrow\{1\} \rightarrow 1 \mathrm{~B})$ \\
2 & $(1 \mathrm{~F} \rightarrow\{2\} \rightarrow 1 \mathrm{~B}-1 \mathrm{~F}-1 \mathrm{~B})$ \\
& $(1 \mathrm{~F}-1 \mathrm{~B}-1 \mathrm{~F}-1 \mathrm{~B}-1 \mathrm{~F} \rightarrow\{1\} \rightarrow 1 \mathrm{~B})(1 \mathrm{~F}-1 \mathrm{~B}-1 \mathrm{~F} \rightarrow\{1\} \rightarrow 1 \mathrm{~B}-1 \mathrm{~F}-1 \mathrm{~B})$ \\
& $(1 \mathrm{~F} \rightarrow\{1\} \rightarrow 1 \mathrm{~B}-1 \mathrm{~F}-1 \mathrm{~B}-1 \mathrm{~F}-1 \mathrm{~B})$ \\
& $(1 \mathrm{~F}-1 \mathrm{~B}-1 \mathrm{~F}-1 \mathrm{~B}-1 \mathrm{~F}-1 \mathrm{~B}-1 \mathrm{~F}-1 \mathrm{~B})$ \\
\hline
\end{tabular}

Table 3. $\{5\}$ : Series of forward and backward steps to return within-range in $(2 n+2)=12$ steps.

\begin{tabular}{cc}
$\begin{array}{c}\text { Number of } \\
\text { visits to origin }\end{array}$ & Sequence of steps \\
\hline 0 & $(1 \mathrm{~F} \rightarrow\{4\} \rightarrow 1 \mathrm{~B})$ \\
1 & $(1 \mathrm{~F}-1 \mathrm{~B}-1 \mathrm{~F} \rightarrow\{3\} \rightarrow 1 \mathrm{~B})(1 \mathrm{~F} \rightarrow\{1\} \rightarrow 1 \mathrm{~B}-1 \mathrm{~F} \rightarrow\{2\} \rightarrow 1 \mathrm{~B})$ \\
& $(1 \mathrm{~F} \rightarrow\{2\} \rightarrow 1 \mathrm{~B}-1 \mathrm{~F} \rightarrow\{1\} \rightarrow 1 \mathrm{~B})(1 \mathrm{~F} \rightarrow\{3\} \rightarrow 1 \mathrm{~B}-1 \mathrm{~F}-1 \mathrm{~B})$ \\
2 & $(1 \mathrm{~F}-1 \mathrm{~B}-1 \mathrm{~F}-1 \mathrm{~B}-1 \mathrm{~F} \rightarrow\{2\} \rightarrow 1 \mathrm{~B})(1 \mathrm{~F}-1 \mathrm{~B}-1 \mathrm{~F} \rightarrow\{1\} \rightarrow 1 \mathrm{~B}-1 \mathrm{~F} \rightarrow\{1\} \rightarrow 1 \mathrm{~B})$ \\
& $(1 \mathrm{~F}-1 \mathrm{~B}-1 \mathrm{~F} \rightarrow\{2\} \rightarrow 1 \mathrm{~B}-1 \mathrm{~F}-1 \mathrm{~B})(1 \mathrm{~F} \rightarrow\{1\} \rightarrow 1 \mathrm{~B}-1 \mathrm{~F}-1 \mathrm{~B}-1 \mathrm{~F} \rightarrow\{1\} \rightarrow 1 \mathrm{~B})$ \\
& $(1 \mathrm{~F} \rightarrow\{1\} \rightarrow 1 \mathrm{~B}-1 \mathrm{~F} \rightarrow\{1\} \rightarrow 1 \mathrm{~B}-1 \mathrm{~F}-1 \mathrm{~B})(1 \mathrm{~F} \rightarrow\{2\} \rightarrow 1 \mathrm{~B}-1 \mathrm{~F}-1 \mathrm{~B}-1 \mathrm{~F}-1 \mathrm{~B})$ \\
& $(1 \mathrm{~F}-1 \mathrm{~B}-1 \mathrm{~F}-1 \mathrm{~B}-1 \mathrm{~F}-1 \mathrm{~B}-1 \mathrm{~F} \rightarrow\{1\} \rightarrow 1 \mathrm{~B})(1 \mathrm{~F}-1 \mathrm{~B}-1 \mathrm{~F}-1 \mathrm{~B}-1 \mathrm{~F} \rightarrow\{1\} \rightarrow 1 \mathrm{~B}-1 \mathrm{~F}-1 \mathrm{~B})$ \\
& $(1 \mathrm{~F}-1 \mathrm{~B}-1 \mathrm{~F} \rightarrow\{1\} \rightarrow 1 \mathrm{~B}-1 \mathrm{~F}-1 \mathrm{~B}-1 \mathrm{~F}-1 \mathrm{~B})(1 \mathrm{~F} \rightarrow\{1\} \rightarrow 1 \mathrm{~B}-1 \mathrm{~F}-1 \mathrm{~B}-1 \mathrm{~F}-1 \mathrm{~B}-1 \mathrm{~F}-1 \mathrm{~B})$ \\
& $(1 \mathrm{~F}-1 \mathrm{~B}-1 \mathrm{~F}-1 \mathrm{~B}-1 \mathrm{~F}-1 \mathrm{~B}-1 \mathrm{~F}-1 \mathrm{~B}-1 \mathrm{~F}-1 \mathrm{~B})$ \\
\hline
\end{tabular}

The above corollary helps us to come up with the following recursive formulation of $\{n\}$ :

$$
\begin{aligned}
& \{n\}=\bigcup_{k=0}^{n}\{n, k\} \\
& \{n, k\}=\bigcup_{i=1}^{n-k}\{i-1\} \rightarrow\{n-i, k-1\} \\
& \{1\}=1 F-1 B \\
& \{0\}=\phi
\end{aligned}
$$

where $k$ denotes the number of times the mobile visits origin and $i$ denotes the first position at which the mobile returns back to origin for a given $k$. Such a formulation does not allow for computation of a closed form expression for $n$. Correspondingly, we formulate an algorithm for determining $\|n\|$ as

Based on Algorithms 1-3, we numerically find the value of Number Of Ways and Total Number Of Ways for 


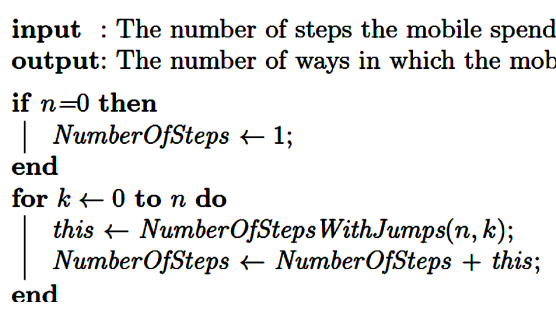

Algorithm 1. Number of steps.

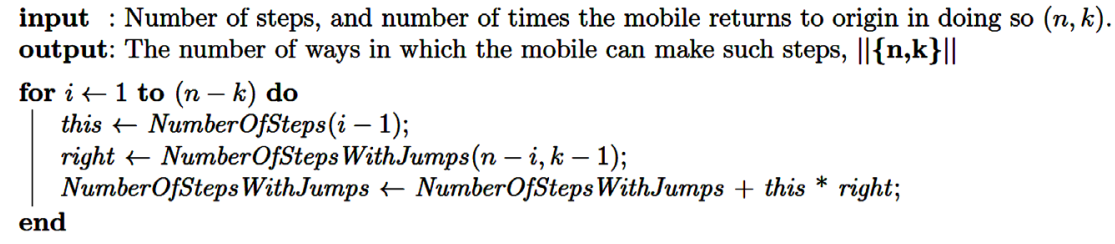

Algorithm 2. Number of steps with jumps.

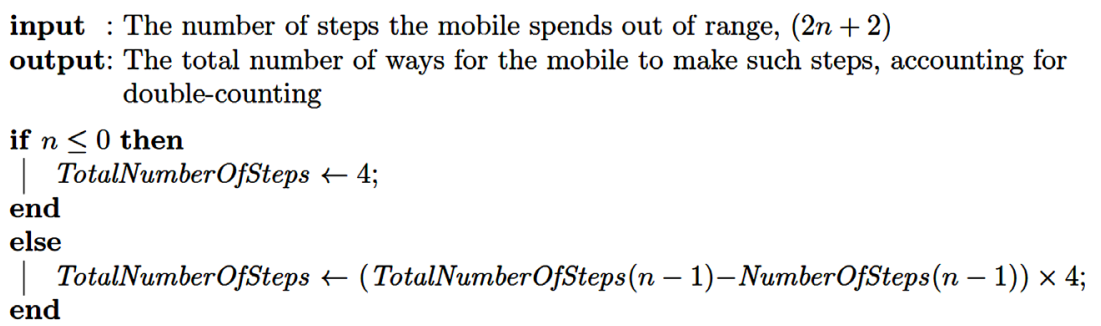

Algorithm 3. Total number of steps.

different number of steps the mobile makes. These are summarized in Table 4.

Equation (31) means that the time $T_{n}$ taken to travel a unit distance at instance $n$ would be distributed as a Gaussian, and that $T_{i}$ and $T_{k}$ would be independent and identically distributed (i.i.d.). Hence, in the 1-D single base-station case, time taken for the mobile to return back to within-range can be written as:

$$
T_{\text {disconnect }}=\left(2 \times \frac{1}{4}+4 \times \frac{1}{12}+6 \times \frac{2}{44}+8 \times \frac{5}{168}+10 \times \frac{14}{652}+12 \times \frac{42}{2552}+\cdots\right) \times T_{n}
$$

The right-hand side of the equation above is plotted in Figure 1. Note that the coefficients in Equation (38) can not be reduced to a closed form, and hence need to be calculated numerically. However, since their values are not dependent on mobility patterns of individual mobile users, these can be easily pre-computed and stored for future use.

While in this paper, we have restricted our analysis to the 1-dimensional single base-station case, the more complicated cases of 1-dimensional multiple base-stations and 2-dimensional multiple base-stations can be solved under the same framework. For example, the 1-dimensional multi-base station case, where the next basestation is designed to be $k$ steps away from the first base-station, can be solved by extending Algorithms 1-3 with the additional constraint that the mobile should not move more than $(k-1)$ steps away from the origin in any intermediate step. The generalized 2-dimensional multiple base-stations case is much more complicated to analyze and is not within the scope of this paper.

\section{Conclusions and Future Work}

In this paper, we looked at disconnection prediction using the Gauss-Markov mobility modeling scheme. This led us to an expression that would be extremely performance intensive for real-world systems. We then developed an alternate mobility model based on Gauss-Markov process. Table 4 represents the number of distinct sequences following which the mobile can land up within range in exactly $(2 n+2)$ steps. These values combined with the distribution of the amount of time the mobile spends between each step of the mobility model 
Table 4. Calculating number of steps and total number of steps for different values of $n$.

\begin{tabular}{cccc}
\hline$n$ & $\|\{n\}\|$ & Total \# of ways & \% of total ways \\
\hline 0 & 1 & 4 & 0.25 \\
1 & 1 & 12 & 0.083333 \\
2 & 2 & 44 & 0.045455 \\
3 & 5 & 168 & 0.029762 \\
4 & 14 & 652 & 0.021472 \\
5 & 42 & 2552 & 0.016458 \\
7 & 429 & 39632 & 0.010825 \\
10 & 16796 & 2466664 & 0.006809 \\
15 & 9694845 & 2457718688 & 0.003945 \\
20 & 6564120420 & 2474716313192 & 0.002652 \\
25 & 4861946401452 & 2504621026560750 & 0.001941 \\
30 & 3814986502092300 & 2542372172343410000 & 0.001501 \\
\hline
\end{tabular}

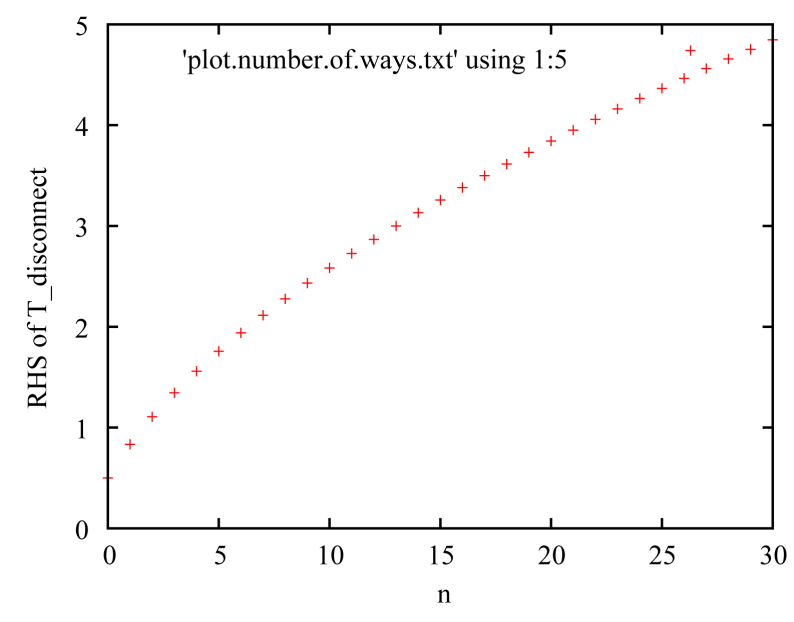

Figure 1. Plot of the r.h.s summation series of Equation (38), showing asymptotically convergent behavior.

helped us get the distribution of the total amount of time spent by the mobile outside range. Such a formulation can then be utilized to serve as the mobility prediction module for various applications and it is not as performance intensive as the one that used the Gauss-Markov mobility model directly.

The computations shown in Table 4 are compute-intensive, and are time-consuming to compute on the fly. However, per Equation (38), the summation series is independent of the nature of movement of a mobile, and hence can be pre-computed and tabulated. Also, the time spent by the mobile in carrying out individual steps $T_{n}$ is also i.i.d., thereby implying that the time spent in $n$ steps would be simply $n$ times the time spent in an individual step.

In this paper, we only looked at the 1-dimensional single base-station case. Future work in this area will involve applying the same mobility model and analysis to the 1-dimensional multiple base-stations case and the 2-dimensional single and multiple base-stations case. In addition, the future research in this area will also look to implement the above disconnection prediction scheme in base-stations to determine its prediction accuracy with real-world data. Finally, extending this scheme to next-generation networks like ad-hoc and wireless sensor networks is another area for future work.

\section{References}

[1] Goff, T., Moronski, J., Phatak, D. S. and Gupta, V. (2000) Freeze-TCP: A True End-to-End TCP Enhancement Me- 
chanism for Mobile Environments. INFOCOM 2000. Annual Joint Conference of the IEEE Computer and Communications Societies. Proceedings, Tel-Aviv, 26-30 March 2000, 10 p.

[2] Bhutani, G. (2010) A Near-Optimal Scheme for TCP ACK Pacing to Maintain Throughput in Wireless Networks. Proceedings of the 2nd International Conference on Communication Systems and Networks, Bangalore, January 2010, 491-497.

[3] Liang, B. and Haas, Z. J. (1999) Predictive Distance-Based Mobility Management for PCS Networks. INFOCOM'99. Proceedings of 18th Annual Joint Conference of the IEEE Computer and Communications Societies, New York, 21-25 March 1999, 1337-1384.

[4] Guerin, R.A. (1987) Channel Occupancy Time Distribution in a Cellular Radio System. IEEE Transactions on Vehicular Technology, 36, 89-99. http://dx.doi.org/10.1109/T-VT.1987.24106

[5] Royer, E.M., Melliar-Smith, P.M. and Moser, L.E. (2001) An Analysis of the Optimum Node Density for ad Hoc Mobile Networks. IEEE International Conference on Communications, Helsinki, 11-14 June 2001, 5 p.

[6] Haas, Z.J. and Pearlman, M.R. (2001) The Performance of Query Control Schemes for the Zone Routing Protocol. IEEE/ACM Transactions on Networking (TON), 9, 427-438. http://dx.doi.org/10.1109/T-VT.1987.24106

[7] Pearlman, M.R., Haas, Z.J., Sholander, P. and Tabrizi, S.S. (2000) On the Impact of Alternate Path Routing for Load Balancing in Mobile Ad Hoc Networks. 2000 1st Annual Workshop on Mobile and Ad Hoc Networking and Computing, Boston, 11 August 2000, 3-10.

[8] Liu, T. and Cerpa, A.E. (2011) Foresee (4C): Wireless Link Prediction Using Link Features. 2011 10th International Conference on Information Processing in Sensor Networks (IPSN), Chicago, 12-14 April 2011, 294-305.

[9] Liu, H., Al-Khafaji, S.K. and Smith, A.E. (2011) Prediction of Wireless Network Connectivity Using a Taylor Kriging Approach. International Journal of Advanced Intelligence Paradigms, 3, 112-121. http://dx.doi.org/10.1504/IJAIP.2011.039744

[10] Konak, A. (2009) A Kriging Approach to Predicting Coverage in Wireless Networks. International Journal of Mobile Network Design and Innovation, 3, 65-71. http://dx.doi.org/10.1504/IJMNDI.2009.030838

[11] Capka, J. and Boutaba, R. (2004) Mobility Prediction in Wireless Networks Using Neural Networks. Management of Multimedia Networks and Services, 3271, 320-333. http://dx.doi.org/10.1007/978-3-540-30189-9 26 